\title{
Happy Birthday, Intensivmedizin up2date!
}

\author{
Hugo Van Aken
}

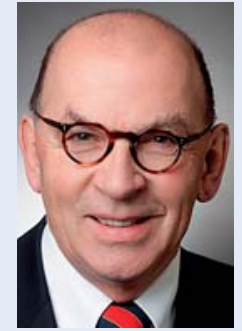

Hugo Van Aken
Es war einmal ... eine erste fixe Idee für eine neue Fachzeitschrift über Intensivmedizin - vor mehr als 10 Jahren. Aber bei den Mengen an Fachartikeln und Fachzeitschriften, die es bereits auf dem Markt gab, musste es etwas Besonderes sein, um sich von den anderen Titeln absetzen und auf dem Markt bestehen zu können. Wir entschieden uns, eine interdisziplinäre Fortbildungszeitschrift für Intensivmediziner verschiedener Fachrichtungen herauszugeben - und das in einem außergewöhnlichen Format. Das Herzstück der Zeitschrift sollten 5 CME-Fachbeiträge sein, die einzeln gebunden und gelocht thematisch sortiert in einem up2date-Sammelordner abgeheftet werden können. Die Idee dabei: Es soll ein sich ständig erneuerndes individuelles Handbuch entstehen. Gesagt, getan: Im Dezember 2004 erschien - pünktlich zum DIVIKongress - die erste Ausgabe der Intensivmedizin up2date - mit fulminantem Erfolg: bereits nach einem Jahr hatten wir über 3000 Abos.

Von Beginn an hatten wir den interdisziplinären Charakter der Zeitschrift klar im Fokus und auf eine ausgewogene Verteilung der Themen geachtet. Auch Arbeiten, die über das rein medizinische Wissen hinausgehen, wie z. B. die Arbeit von Czeschinski et al. „Leben und Arbeiten im Schichtdienst“ aus dem letzten Heft, finden regelmäßig Eingang in den Themenplan.

Die notfallmedizinische Rubrik wurde nach 3 Jahren eingestellt. Aus ihr entstand die neue up2date-Zeitschrift „Notfallmedizin up2date“, die inzwischen auch seit knapp 8 Jahren auf dem Markt ist. Wir möchten an dieser Stelle dem damaligen Rubrikherausgeber,

Wir haben Euch alles gelehrt, was wir können, wir haben Euch alles beigebracht, was wir wissen,

aber bitte denkt immer daran,

dass zukünftige Ereignisse wahrscheinlich beweisen werden,

dass die Hälfte dessen,

was wir Euch gelehrt haben, falsch ist -

leider wissen wir nicht, welche Hälfte".

R. Dillemans, 1987

Rektor der Katholischen Universität Leuven Belgien (1986-1998)
Herrn Professor Bernd Böttiger, noch einmal ganz herzlich für sein Engagement danken.

Apropos Dank: Danken wollen wir an dieser Stelle auch ganz besonders Herrn Professor Werner Hacke. Er war seit der Gründung der Zeitschrift als Herausgeber aktiv und hat die Zeitschrift und seine neurologische Rubrik ganz wesentlich gestaltet und geprägt. Er scheidet nun nach insgesamt 10 Jahren aus dem Herausgeberboard aus und übergibt seine Aufgaben bei der Intensivmedizin up2date an Herrn Professor Kollmar für die neurologischen Themen und Herrn Professor Unterberg für den Bereich Neurochirurgie. Ein herzliches Willkommen den beiden Neuen!

Und was hat sich am Heft selbst in den letzten 10 Jahren getan - abgesehen von der bunten Themenmischung der CME-Beiträge? Der sogenannte „Mantelteil“ ist zum festen Bestandteil unserer Zeitschrift geworden. Doch was verbirgt sich dahinter? Zum einen die Rubrik „Für Sie gelesen“ mit Zusammenfassungen nationaler und internationaler Studien - koordiniert durch Herrn Professor Bone als Rubrikherausgeber, der für die treffende Auswahl der Studien und die lehrreichen Zusammenfassungen viel positive Leserrückmeldung bekommt. Zum anderen die kürzlich neu etablierte Rubrik „SOPs in der Intensivmedizin“ - praxisnah und nützlich für alle Kliniker und mit den beiden Rubrikherausgebern Herrn Professor Ellger und Herrn Dr. Bösel in sehr kompetenten Händen. Und last but not least das Editorial, in dem wir immer wieder brandaktuelle oder auch kontroverse Themen aufgreifen und diskutieren - ohne Scheu.

Und wie geht es nun weiter? Unser explizites Ziel ist es, auch weiterhin so vielfältig, aktuell und kliniknah zu bleiben, damit wir auch zum 20-jährigen Jubiläum sagen können: „Der Weg ist richtig!“

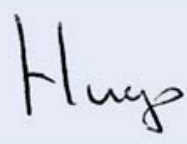

